\title{
Monte Carlo simulations for the Pierre Auger Observatory using the VO auger grid resources
}

\author{
Eva Santos ${ }^{a, *}$ on behalf of the Pierre Auger $^{b}$ Collaboration \\ (a complete list of authors can be found at the end of the proceedings) \\ ${ }^{a}$ Institute of Physics of the Czech Academy of Sciences, Prague, Czech Republic \\ ${ }^{b}$ Observatorio Pierre Auger, Av. San Martín Norte 304, 5613 Malargüe, Argentina \\ E-mail: spokespersons@auger.org
}

The Pierre Auger Observatory, located near Malargüe, Argentina, is the world's largest cosmic-ray detector. It comprises a $3000 \mathrm{~km}^{2}$ surface detector and 27 fluorescence telescopes, which measure the lateral and longitudinal distributions of the many millions of air-shower particles produced in the interactions initiated by a cosmic ray in the Earth's atmosphere. The determination of the nature of cosmic rays and studies of the detector performances rely on extensive Monte Carlo simulations describing the physics processes occurring in extensive air showers and the detector responses.

The aim of the Monte Carlo simulations task is to produce and provide the Auger Collaboration with reference libraries used in a wide variety of analyses. All multipurpose detector simulations are currently produced in local clusters using Slurm and HTCondor. The bulk of the shower simulations are produced on the grid, via the Virtual Organization auger, using the DIRAC middleware. The job submission is made via python scripts using the DIRAC-API.

The Auger site is undergoing a major upgrade, which includes the installation of new types of detectors, demanding increased simulation resources. The novel detection of the radio component of extensive air showers is the most challenging endeavor, requiring dedicated shower simulations with very long computation times, not optimized for the grid production.

For data redundancy, the simulations are stored on the Lyon server and the grid Disk Pool Manager and are accessible to the Auger members via iRODS and DIRAC, respectively. The CERN VMFile System is used for software distribution where, soon, the Auger $\overline{\text { Offlline }}$ software will also be made available.

$37^{\text {th }}$ International Cosmic Ray Conference (ICRC 2021)

July 12th - 23rd, 2021

Online - Berlin, Germany

\footnotetext{
${ }^{*}$ Presenter
} 


\section{Introduction}

Cosmic rays are mostly fully ionized atomic nuclei that permanently reach us from outside the Solar system. More than one century has passed since their discovery [1]. However, their nature, sources, and acceleration mechanisms are still largely unknown.

The cosmic-ray spectrum can be relatively well described over an extensive energy range by a power-law whose spectral index slightly deviates from 3 , showing remarkably very few distinctive features [2]. Whereas at sub-GeV energies, the mass composition of cosmic rays can be measured directly by balloon or space-borne experiments with isotope precision, and at multi- $\mathrm{TeV}$ with elemental precision [3, 4], significant uncertainties rapidly arise as soon as direct detections cease to be viable. Coincidently, around the same energy, the indirect detection of cosmic rays becomes feasible thanks to the many millions of particles generated in a cascading process that initiates from the interaction of the cosmic ray in the Earth's atmosphere - a phenomenon called an extensive air shower. In the latter, the cosmic-ray energy and mass composition have to be derived indirectly from air shower and detector simulations.

However, at the highest energies, full shower simulations become computationally prohibitive since the number of secondary particles produced in one extensive air shower, which scales linearly with the energy of the primary cosmic ray, exceeds $10^{10}$ at $10^{19} \mathrm{eV}[5,6]$. These limitations were overcome by the development of thinning algorithms, which allow reducing the number of simulated particles with energies falling below a pre-defined threshold [7]. In this case, only a representative fraction of the particles is simulated, and a weight indicating the number of 'thinned' particles is assigned. More recently, CPU (Central Process Unit) time-intensive air shower simulations, such as the ones of showers without thinning, or those including the radio emission or the Cherenkov light, became more accessible thanks to the parallelization of the Monte Carlo codes, see for instance [8].

Some Monte Carlo codes implementing the features described above are CORSIKA [9] and AIRES [10]. Other codes which treat the particle transport and interactions with the medium used to simulate the detector response are, for example, FLUKA [11, 12] and GEANT4 [13].

The interpretation of some properties of cosmic rays requires extensive simulation libraries that can only be produced using local computing farms or grid computation.

\section{Pierre Auger Observatory}

Designed to study the most energetic particles in the Universe, the Pierre Auger Observatory is the world's largest cosmic-ray detector. It is located near the Argentinian city of Malargüe, province of Mendoza, at a mean altitude of $\sim 1400 \mathrm{~m}$ above sea level, corresponding to an atmospheric overburden of $\sim 875 \mathrm{~g} \mathrm{~cm}^{-2}$. It is a hybrid detector, which combines a Surface Detector (SD) array extending over a $3000 \mathrm{~km}^{2}$ area with a Fluorescence Detector (FD), comprising four sites at the array periphery, housing a total of 27 fluorescence telescopes. A detailed description of the Observatory can be found in [14].

\subsection{AugerPrime}

In operation since 2004, the Pierre Auger Observatory was completed in 2008. Currently, the Auger site is undergoing a major upgrade, called AugerPrime, which consists of an enhancement of its SD stations, along with an extension of the FD duty cycle from $15 \%$ to about $30 \%$. 
The main objective of AugerPrime is to improve the determination of the mass composition of the highest energy cosmic rays with the SD array on an event-by-event basis. Such measurements will allow us to understand the origin of the flux suppression and determine the fraction of light elements at the highest energies. Also, the potential of studying multi-particle physics at the $\sqrt{s} \sim 100 \mathrm{TeV}$ scale will be pursued $[15,16]$.

The key feature towards an improved mass composition with the SD is to disentangle the electromagnetic and muonic signals measured by the water-Cherenkov detectors. Hence, a surface scintillator detector (SSD) and a radio detector (RD) are being installed on top of the SD stations. Both detectors are complementary since the SSDs are mostly sensitive to showers with zenith angles up to $45^{\circ}$, while the RD works better for air showers with zenith angles above $60^{\circ}$. In Figure 1 , schematic sketches of the Pierre Auger Observatory (left) and the AugerPrime detector stations (right) are shown. Additionally, an electronics board capable of connecting all the detectors, with
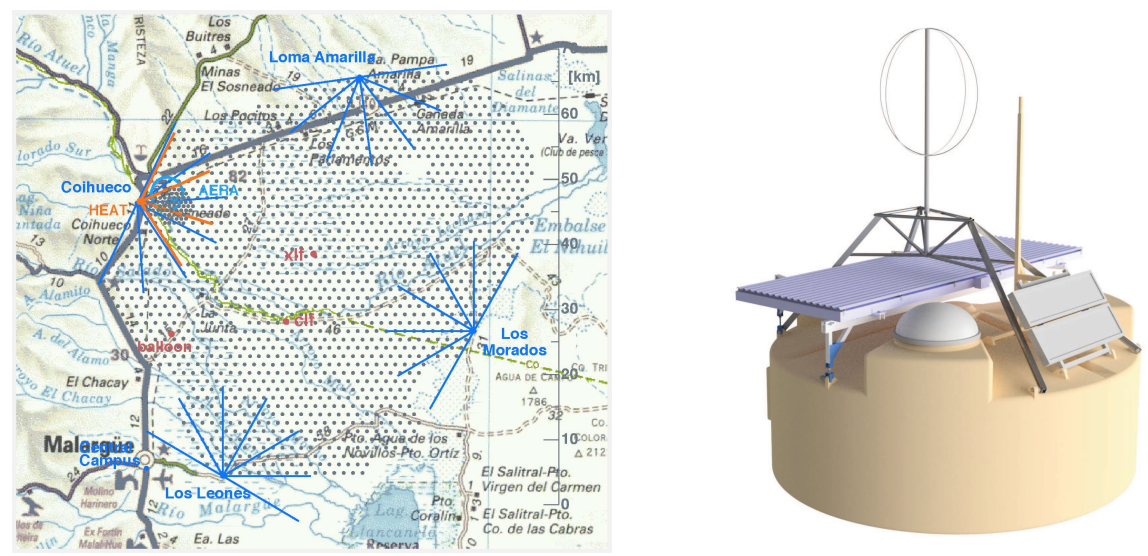

Figure 1: Left: Schematic sketch of the Pierre Auger Observatory. Right: Schematic sketch of an AugerPrime station with the SSD and RD mounted on top of the water-Cherenkov detector.

3 times higher time resolution, and the installation of a small PMT inside the tank, will allow measuring the lateral distribution of the shower particles as close as $250 \mathrm{~m}$ from the shower axis, improving the event reconstruction. Finally, underneath an area of $25 \mathrm{~km}^{2}$ within the Observatory, an underground muon detector operating in the energy range $10^{17.5}-10^{19} \mathrm{eV}$, will allow a direct measurement of the muon component with $E_{\mu}>1 \mathrm{GeV}$ for showers with zenith angles below $45^{\circ}[17]$.

\section{Virtual Organization auger}

The VO auger was created in 2006 by the Czech group in cooperation with the CESNET Metacentrum [18-20]. The CESNET Metacentrum provides and maintains the central resources such as the registration portal and the VOMS (Virtual Organization Membership Service) server.

All members of the Pierre Auger Collaboration can apply for membership by filling a registration form which has to be approved by the VO manager. The membership has a validity of one year, after which it can be renewed under request. As of May 2021, the VO auger had 32 individual members. 
The VO auger comprises 23 grid sites in 11 countries. More than 1.3 million files are registered in the DIRAC File Catalog; these files are stored on 13 different sites and occupy 210 TB of disk space from a total of 1.4 PB storage capacity.

\subsection{DIRAC interware}

In 2014 the VO auger adopted the DIRAC (Distributed Infrastructure with Remote Agent Control) interware for the job submission, monitoring, and file catalog management [21, 22]. The DIRAC server, at https://dirac.france-grilles.fr/DIRAC/, runs on the France Grilles Infrastructure [23]. Its usage only requires having a running DIRAC client installed. A client version is provided in the DIRAC CVMFS [24] repository dirac . egi . eu (see Section 4).

\subsection{Grid computing in 2020}

According to the EGI - European Grid Infrastructure accounting portal [25], in 2020, the VO auger ran nearly $10^{5}$ single-core jobs which used more than 60 million hours (normalized elapsed time). The jobs ran in 6 grid sites from 5 different regions. The bulk of the jobs, which had a stringent requirement of $10^{7} \mathrm{CPU}$ seconds walltime, the maximum allowed limit, may greatly justify the small number of sites accepting our jobs. The production started to ramp up in the second quarter of the year, reaching an average of 1000 used cores in the second semester, primarily due to the CoREAS [26] jobs for the work presented in [27]. When excluding the LHCb, pheno, and Fermilab groups' contributions, which we do not consider as belonging to the Astrophysics category, the VO auger used an amount of normalized walltime comparable to other Astrophysics VOs (see Figure 2).

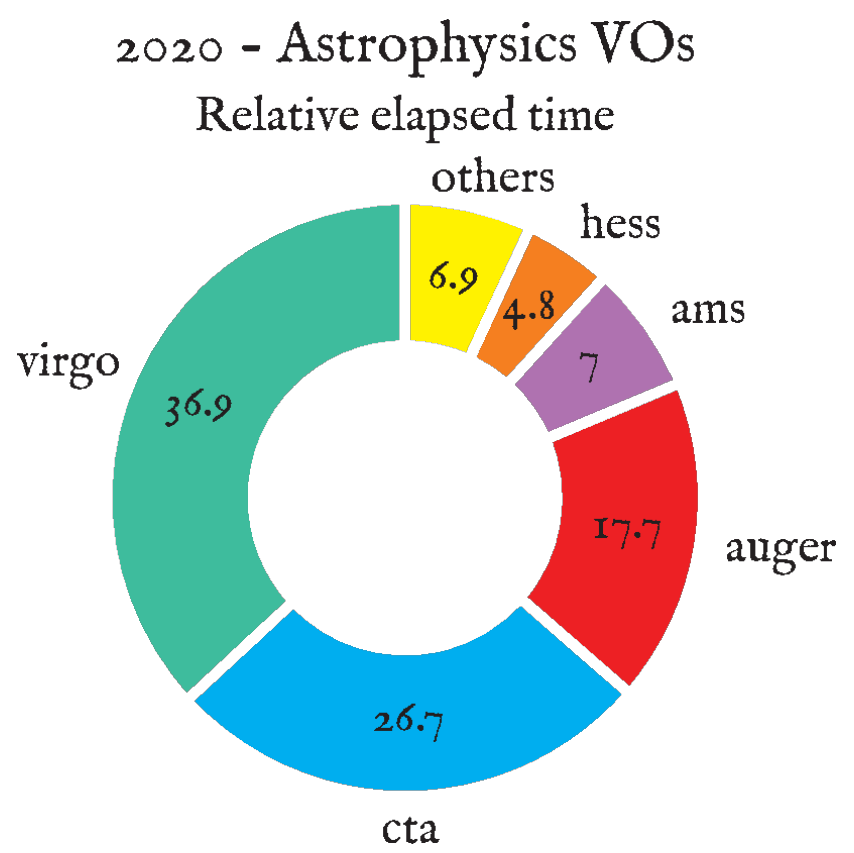

Figure 2: Relative elapsed time of the Astrophysics VOs in 2020, according to the EGI accountig portal [25]. The contributions of the LHCb, pheno, and Fermilab groups were excluded. 


\section{Monte Carlo simulations Task}

The main objectives of the Monte Carlo simulations task are to produce multipurpose reference air shower and detector simulation libraries, see [28-30] for some usage examples, and also extensive and CPU-intensive simulations when requested, as in [27]. The task also collaborates closely with

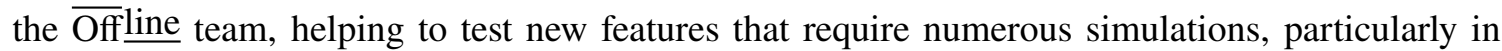
relatively short time scales. Below, a description of the computing resources, production framework, and multipurpose simulation libraries are given.

\subsection{Computing resources}

The data storage requirements for the whole set of Auger data and simulations currently amounts from several to many hundreds of TB, a reasonably modest output compared to other high-energy physics (HEP) experiments.

The total volume dedicated to Monte Carlo simulations undoubtedly comprises the bulk of disk storage. However, its actual dimension is hard to determine since it is scattered among many groups' computing farms and in the grid Disk Pool Manager (DPM), consult [31] for the full list. In particular, the Monte Carlo Simulations task alone has produced more than $200 \mathrm{~TB}$ only in grid productions.

CORSIKA files, which have typical outputs of several hundreds of MB, occupy the most significant fraction of the storage volume. For redundancy, in the most recent productions, all files are stored in the IN2P3 Computing Center in Lyon [32], where all Auger members can access them via iRODS - integrated Rule-Oriented Data System [33].

In its turn, the total data volume required to store all data produced at the Pierre Auger Observatory is of the order of $50 \mathrm{~TB}$. These data are divided into three main categories: raw, monitoring, and $\overline{\text { Offline }}$, i.e., high-level data typically used in all the physics analyses [34]. All raw data, comprising about $46 \mathrm{~TB}$, are stored locally in Malargüe and mirrored every 3 hours to the IN2P3 Computing Center in Lyon. Monitoring data accounts for $\sim 5 \mathrm{~TB}$, and are mirrored at different sites. Finally, all data processed with the Offline framework [34], namely, raw data and simulations, comprises the tiniest fraction of disk space since typical file sizes are of the order of $1 \mathrm{MB}$.

\subsection{CERN Virtual Machine - File System}

The CERN Virtual Machine - File System (CVMFS) provides a scalable, reliable, and lowmaintenance software distribution service [24]. It aims to provide a complete and portable environment for running LHC and HEP data analysis on any end-user computer and the grid, independently of the operating system platforms. Each user community defines the procedures for building, installing, and validating software [35].

Auger has its own CVMFS repository, under the name of auger.egi .eu, where its relevant software is installed. Only CORSIKA is currently available. There are ongoing efforts to have an $\overline{\mathrm{Off}}$ line installation in the Auger CVMFS repository soon. In our setup, the CVFMS is essentially used to run the task's jobs, providing a uniform configuration that can be used in a heterogeneous network of local computing farms and the grid. As soon as the $\overline{\mathrm{Offl}}$ line becomes available in the CVMFS, the bulk of the productions will be transferred to the grid, leaving the local computing farms free for processing CPU-time demanding jobs that cannot run on the grid mainly due to walltime limitations. 


\subsection{Production framework}

Efforts are being made towards creating a uniform environment across all Auger computing systems. CentOS7, from Community Enterprise Operating System, is the chosen operating system [36]. Systems must also have CVMFS [24] installed for software distribution, HTCondor [37] for local job submission, and the DIRAC server [21] for grid usage.

Currently, there is no centralized system to submit jobs to the local computing farms. Each group has its own set of scripts, or programs, tailored for their own use.

\subsubsection{Grid production}

In the task, a bundle of Python 2.7 scripts is used to submit jobs to the grid, using the DIRAC API (Application Programming Interface) functionalities, which are easily adapted to run jobs in the local computing farms using HTCondor or Slurm. These scripts will be readily available for sharing within the Collaboration. CORSIKA [9] and AIRES [10] scripts for DIRAC, HTCondor, and Slurm currently exist.

The bulk of the grid jobs uses this Python script bundle since a small team runs a fraction of the productions. Thus, homogeneity is a requirement for the consistency of the simulations. All jobs consist of only one CORSIKA file per job since typical run times can vary from several hours to more than one week if the radio component of air showers is activated.

\subsubsection{Local computing farm production}

For illustration, we give examples of the submission of jobs in local computing farms. In some cases, a $\mathrm{C}++$ program is used to submit CORSIKA and $\overline{\mathrm{Offl}}$ line jobs through the batch system Slurm. This program receives as input a set of parameters which allow to steer the CORSIKA and

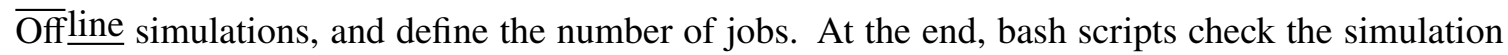
outputs and resubmit the failed jobs.

Instead, other groups typically use bash or python scripts to handle their local production. Recently, CORSIKA jobs use the recent Python 2.7 script bundle adapted from DIRAC to HTCondor,

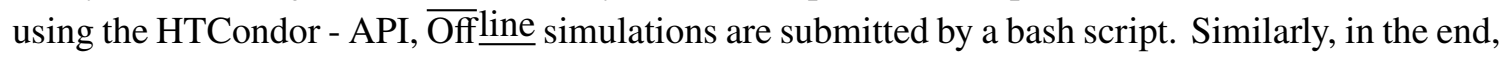
according to the type of simulation, Python 2.7 or bash scripts check the integrity of the output files and recover the failed simulations. For $\overline{\mathrm{Off}} \underline{\mathrm{line}}$ simulations, each batch also includes the application of standard FD and SD event selection criteria and production of accompanying documentation with the plots characterizing the performances of the reconstruction, which is placed to a dedicated wikipage of the library.

While standard CORSIKA simulations have CPU memory requirements of the order of $1 \mathrm{~GB}$ if FLUKA $[11,12]$ is used to simulate the low-energy hadronic interactions, typical $\overline{\text { Offline jobs }}$ require several hundreds of MB of memory and less than one hour of processing time.

\subsection{Reference simulation libraries}

The reference simulation libraries consist of CORSIKA [9] extensive air shower simulations later injected into $\overline{\mathrm{Off}}$ line [34], where the simulation of the detector's response and event reconstruction occurs. The libraries are continuously updated, and several CORSIKA and Offline versions were used over the years.

Per CORSIKA version, three high-energy hadronic interaction models are simulated: EPOSLHC [38, 39], QGSJetII-0.4 [40, 41], and Sibyll 2.3* [42-44], and four hadronic species, namely: 
Hydrogen, Helium, Oxygen, and Iron. FLUKA [11, 12] is used to treat elastic and inelastic hadronic interactions below $80 \mathrm{GeV}$ in air. The library comprises air shower simulations ranging from $10^{15}-10^{20.2} \mathrm{eV}$, with zenith angles below $65^{\circ}$ uniformly distributed in $\sin \theta \cos \theta$, arranged in energy bins of width $\log _{10}(E / \mathrm{eV})=0.5$, except for the highest energy bin, with a spectral index of -1 . There are 5000 showers per bin simulated with an optimal thinning [9] of $10^{-6}$. Up to 4 different Malargüe atmospheres can be found in the simulations.

Finally, there are plenty of simulations for photon primaries with pre-shower activated for energies above $10^{19} \mathrm{eV}$. The standard photon simulations follow a similar structure as the one described above. In this case, there are 10000 showers per energy bin.

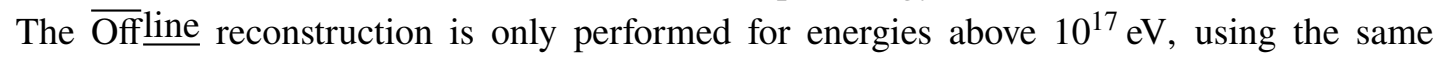

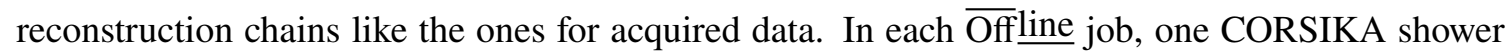
is processed by throwing it multiple times at random positions of the SD array. To increase the number of reconstructed events, the number of resamplings varies from 5 to 30 , depending on the energy of the primary particle.

All Auger data and a subset of the simulations produced are stored in the IN2P3 Computing Center in Lyon, France. iRODS is the preferred system to download simulations from the Lyon Computing Center [33]. All the task's simulations and many others produced by several Auger groups are shared with the whole Collaboration this way.

\section{Next productions}

In the dawn of the multi-messenger era and on the advent of AugerPrime, the production of two major simulation libraries is about to start. These are the production of reference neutrino libraries for neutral and charged-current interaction modes in all Auger detection channels, accompanied by a library of inclined air showers with zenith angles ranging from $50^{\circ}<\theta<89^{\circ}$ in which the radio component of air showers is included.

Studies to run CORSIKA jobs using Message Passing Interface (MPI) are ongoing since most grid sites do not accept jobs that take longer than three days. Also, in the future, we plan to start our CORSIKA 8 simulation libraries [45].

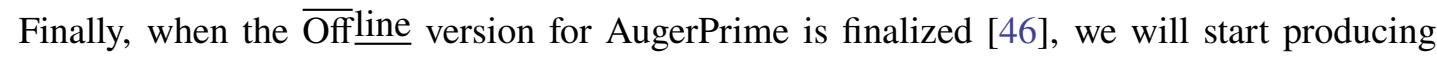
reference $\overline{\mathrm{Off}}$ line libraries for AugerPrime.

\section{Conclusions}

AugerPrime progresses at a good pace, and some data is already being acquired, from which updated reference libraries are very much required.

Simulations for the radio component of air showers pose many challenges due to the long CPU time required to simulate a single shower. Therefore, we are studying the possibility of using the parallelization of the CORSIKA code [8] to provide enough simulations for the radio studies and see if it can be used in the grid.

The grid usage in 2020 doubled when compared with the previous year. We aim to increase the simulation budget this year with the two new productions.

\section{Acknowledgements}

This work is funded by the Czech Republic grants of MEYS CR: LTT18004, LM2018102, CZ.02.1.01/0.0/0.0/18_046/0016010 and GAC̆R 21-02226M. 


\section{References}

[1] V. Hess, Phys. Z. 13 (1912) 1084-1091; V. Hess, (2018), [1808. 02927].

[2] J. Blümer, R. Engel, and J. R. Hörandel, Prog. Part. Nucl. Phys. 63 (2009) 293-338, [0904. 0725].

[3] K. Lodders, Astrophys. J. 591 (2003) 1220-1247.

[4] M. Aguilar et al. [AMS Coll.], Phys. Rept. 894 (2021) 1-116.

[5] J. Matthews, Astropart. Phys. 22 (2005) 387-397.

[6] R. Engel, D. Heck, and T. Pierog, Ann. Rev. Nucl. Part. Sci. 61 (2011) 467-489.

[7] A. M. Hillas, Nucl. Phys. B Proc. Suppl. 52 (1997) 29-42.

[8] T. Pierog et al., Proc. 34th Int. Cosmic Ray Conf., the Hague, the Netherlands (2015), PoS(2015)528.

[9] D. Heck, J. N. Capdevielle, G. Schatz, and T. Thouw, "CORSIKA: A Monte Carlo Code to Simulate Extensive Air Showers", Report FZKA 6019, Forschungszentrum Karlsruhe.

[10] S. J. Sciutto, "AIRES: A system for air shower simulations", (1999) , [astro-ph/9911331].

[11] A. Ferrari, P. R. Sala, A. Fasso, and J. Ranft, "FLUKA: A multi-particle transport code", (2005) .

[12] G. Battistoni et al., Ann. Nucl. Energy 82 (2015) 10-18.

[13] S. Agostinelli et al., Nucl. Instrum. Meth. A 506 (2003) 250-303.

[14] A. Aab et al. [Pierre Auger Coll.], Nucl. Instrum. Meth. A 798 (2015) 172-213, [1502 .01323].

[15] A. Aab et al. [Pierre Auger Coll.], (2016) , [1604.03637].

[16] A. Castellina [for the Pierre Auger Coll.], EPJ Web Conf. 210 (2019) 06002, [1905 . 04472].

[17] F. Sánchez [for the Pierre Auger Coll.], Proc. 36th Int. Cosmic Ray Conf., Madison, Wisconsin, USA (2019), PoS(ICRC2019)411, [1909.09073].

[18] https://www.metacentrum.cz/en/vo/auger/.

[19] https://www. cesnet.cz/.

[20] J. Chudoba et al. [for the Pierre Auger Coll.], J. Phys. Conf. Ser. 219 (2010) 072033

[21] http://diracgrid.org/.

[22] J. Chudoba [for the Pierre Auger Coll.], J. Phys. Conf. Ser. 664 (2015) 032005.

[23] https://dirac.france-grilles.fr/.

[24] https://cernvm.cern.ch/fs/.

[25] https://accounting.egi.eu/.

[26] T. Huege, M. Ludwig, and C. W. James, AIP Conf. Proc., 1535 (2013) 128, [1301.2132].

[27] B. Pont [for the Pierre Auger Coll.], these proceedings, PoS(ICRC2021)387.

[28] J. Vícha [for the Pierre Auger Coll.], these proceedings, PoS(ICRC2021)310.

[29] J. Carceller [for the Pierre Auger Coll.], these proceedings, PoS(ICRC2021)229.

[30] J. Glombitza [for the Pierre Auger Coll.], these proceedings, PoS(ICRC2021)359.

[31] https://www .auger .org/science/publications/icrc-papers/276-computational-resources

[32] https://cc.in2p3.fr/.

[33] https://irods.org/.

[34] S. Argirò et al., Nucl. Instrum. Meth. A 580 (2007) 1485-1496, [0707.1652].

[35] P. Buncic et al., J. Phys. Conf. Ser., 219 (2010) 042003.

[36] https://www. centos.org/, https://www.redhat.com/en/blog/community-profile-centos-project.

[37] https://research.cs.wisc.edu/htcondor/.

[38] K. Werner, F.-M. Liu, and T. Pierog, Phys. Rev. C 74 (2006) 044902, [hep-ph/0506232].

[39] T. Pierog, I. Karpenko, J. M. Katzy, E. Yatsenko, and K. Werner, Phy. Rev. C 92 (2015) 034906, [1306. 0121$].$

[40] S. Ostapchenko, Nucl. Phys. B Proc. Suppl. 151 (2006) 143-146, [hep-ph/0412332].

[41] S. Ostapchenko, Phys. Rev. D 83 (2011) 014018, [1010. 1869].

[42] R. Fletcher, T. Gaisser, P. Lipari, and T. Stanev, Phys. Rev. D 50 (1994) 5710-5731.

[43] F. Riehn, R. Engel, A. Fedynitch, T. K. Gaisser, and T. Stanev, Proc. 34th Int. Cosmic Ray Conf., the Hague, the Netherlands (2015), PoS(ICRC2015)236, [1510.00568].

[44] R. Engel, A. Fedynitch, T. K. Gaisser, F. Riehn, and T. Stanev, Phys. Rev. D 102 (2020) 063002, [1912 . 03300].

[45] A. Alves Junior [for the CORSIKA-8 Coll.], these proceedings, PoS(ICRC2021)284.

[46] L. Nellen [for the Pierre Auger Coll.], these proceedings, PoS(ICRC2021)250. 


\section{The Pierre Auger Collaboration}

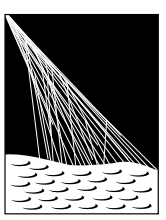

PIERRE

AUSGERVATORY

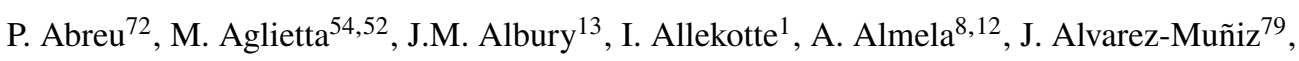
R. Alves Batista ${ }^{80}$, G.A. Anastasi ${ }^{63,52}$, L. Anchordoqui ${ }^{87}$, B. Andrada ${ }^{8}$, S. Andringa ${ }^{72}$, C. $\mathrm{Aramo}^{50}$, P.R. Araújo Ferreira ${ }^{42}$, J. C. Arteaga Velázquez ${ }^{67}$, H. Asorey $^{8}$, P. Assis ${ }^{72}$, G. Avila ${ }^{11}$, A.M. Badescu ${ }^{75}$, A. Bakalova ${ }^{32}$, A. Balaceanu ${ }^{73}$, F. Barbato ${ }^{45,46}$, R.J. Barreira Luz $^{72}$, K.H. Becker ${ }^{38}$, J.A. Bellido ${ }^{13,69}$, C. Berat ${ }^{36}$, M.E. Bertaina ${ }^{63,52}$, X. Bertou ${ }^{1}$, P.L. Biermann ${ }^{b}$, V. Binet ${ }^{6}$, K. Bismark ${ }^{39,8}$, T. Bister ${ }^{42}$, J. Biteau ${ }^{37}$, J. Blazek ${ }^{32}$, C. Bleve ${ }^{36}$, M. Boháčová ${ }^{32}$, D. Boncioli ${ }^{57,46}$, C. Bonifazi ${ }^{9,26}$, L. Bonneau Arbeletche ${ }^{21}$, N. Borodai ${ }^{70}$, A.M. Botti ${ }^{8}$, J. Brack ${ }^{d}$, T. Bretz ${ }^{42}$, P.G. Brichetto Orchera ${ }^{8}$, F.L. Briechle ${ }^{42}$, P. Buchholz ${ }^{44}$, A. Bueno ${ }^{78}$, S. Buitink ${ }^{15}$, M. Buscemi ${ }^{47}$, M. Büsken ${ }^{39,8}$, K.S. Caballero-Mora ${ }^{66}$, L. Caccianiga ${ }^{59,49}$, F. Canfora ${ }^{80,81}$, I. Caracas ${ }^{38}$, J.M. Carceller ${ }^{78}$, R. Caruso ${ }^{58,47}$, A. Castellina ${ }^{54,52}$, F. Catalani ${ }^{19}$, G. Cataldi ${ }^{48}$, L. Cazon ${ }^{72}$, M. Cerda ${ }^{10}$, J.A. Chinellato ${ }^{22}$, J. Chudoba ${ }^{32}$, L. Chytka ${ }^{33}$, R.W. Clay ${ }^{13}$, A.C. Cobos Ceruttii ${ }^{7}$, R. Colalillo ${ }^{60,50}$, A. Coleman ${ }^{93}$, M.R. Coluccia ${ }^{48}$, R. Conceição ${ }^{72}$, A. Condorelli ${ }^{45,46}$, G. Consolati ${ }^{49,55}$, F. Contreras ${ }^{11}$, F. Convenga ${ }^{56,48}$, D. Correia dos Santos $^{28}$, C.E. Covault ${ }^{85}$, S. Dasso ${ }^{5,3}$, K. Daumiller ${ }^{41}$, B.R. Dawson ${ }^{13}$, J.A. Day ${ }^{13}$, R.M. de Almeida $^{28}$, J. de Jesús ${ }^{8,41}$, S.J. de Jong ${ }^{80,81}$, G. De Mauro ${ }^{80,81}$, J.R.T. de Mello Neto ${ }^{26,27}$, I. De Mitri ${ }^{45,46}$, J. de Oliveira ${ }^{18}$, D. de Oliveira Franco ${ }^{22}$, F. de Palma ${ }^{56,48}$, V. de Souza $^{20}$, E. De Vito ${ }^{56,48}$, M. del Río ${ }^{11}$, O. Deligny ${ }^{34}$, L. Deval ${ }^{41,8}$, A. di Matteo $^{52}$, C. Dobrigkeit ${ }^{22}$, J.C. D’Olivo ${ }^{68}$, L.M. Domingues Mendes ${ }^{72}$, R.C. dos Anjos ${ }^{25}$, D. dos Santos $^{28}$, M.T. Dova ${ }^{4}$, J. Ebr ${ }^{32}$, R. Engel ${ }^{39,41}$, I. Epicoco ${ }^{56,48}$, M. Erdmann ${ }^{42}$, C.O. Escobar ${ }^{a}$, A. Etchegoyen ${ }^{8,12}$, H. Falcke ${ }^{80,82,81}$, J. Farmer ${ }^{92}$, G. Farrar ${ }^{90}$, A.C. Fauth ${ }^{22}$, N. Fazzini ${ }^{a}$, F. Feldbusch ${ }^{40}$, F. Fenu ${ }^{54,52}$,

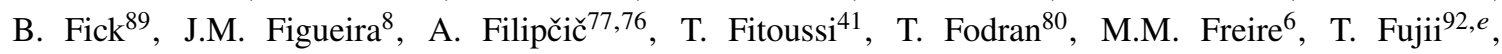
A. Fuster $^{8,12}$, C. Galea ${ }^{80}$, C. Galelli ${ }^{59,49}$, B. García ${ }^{7}$, A.L. Garcia Vegas ${ }^{42}$, H. Gemmeke ${ }^{40}$, F. Gesualdi ${ }^{8,41}$, A. Gherghel-Lascu ${ }^{73}$, P.L. Ghia ${ }^{34}$, U. Giaccari ${ }^{80}$, M. Giammarchi ${ }^{49}$, J. Glombitza ${ }^{42}$, F. Gobbi ${ }^{10}$, F. Gollan ${ }^{8}$, G. Golup ${ }^{1}$, M. Gómez Berisso ${ }^{1}$, P.F. Gómez Vitale ${ }^{11}$, J.P. Gongora ${ }^{11}$, J.M. González ${ }^{1}$, N. González ${ }^{14}$, I. Goos ${ }^{1,41}$, D. Góra ${ }^{70}$, A. Gorgi ${ }^{54,52}$, M. Gottowik ${ }^{38}$, T.D. Grubb ${ }^{13}$, F. Guarino ${ }^{60,50}$, G.P. Guedes ${ }^{23}$, E. Guido ${ }^{52,63}$, S. $\mathrm{Hahn}^{41,8}$, P. $\mathrm{Hamal}^{32}$, M.R. Hampel ${ }^{8}$, P. Hansen ${ }^{4}$, D. Harari ${ }^{1}$, V.M. Harvey ${ }^{13}$, A. Haungs ${ }^{41}$, T. Hebbeker ${ }^{42}$, D. Heck ${ }^{41}$, G.C. Hill ${ }^{13}$, C. Hojvat ${ }^{a}$, J.R. Hörandel ${ }^{80,81}$, P. Horvath ${ }^{33}$, M. Hrabovský ${ }^{33}$, T. Huege ${ }^{41,15}$, A. Insolia ${ }^{58,47}$, P.G. Isar $^{74}$, P. Janecek ${ }^{32}$, J.A. Johnsen ${ }^{86}$, J. Jurysek ${ }^{32}$, A. Kääpä ${ }^{38}$, K.H. Kampert ${ }^{38}$, N. Karastathis ${ }^{41}$, B. Keilhauer ${ }^{41}$, J. Kemp ${ }^{42}$, A. Khakurdikar ${ }^{80}$, V.V. Kizakke Covilakam $^{8,41}$, H.O. Klages ${ }^{41}$, M. Kleifges ${ }^{40}$, J. Kleinfeller ${ }^{10}$, M. Köpke ${ }^{39}$, N. Kunka ${ }^{40}$, B.L. Lago ${ }^{17}$, R.G. Lang ${ }^{20}$, N. Langner ${ }^{42}$, M.A. Leigui de Oliveira ${ }^{24}$, V. Lenok $^{41}$, A. Letessier-Selvon ${ }^{35}$, I. LhenryYvon $^{34}$, D. Lo Presti ${ }^{58,47}$, L. Lopes ${ }^{72}$, R. López ${ }^{64}$, L. Lu ${ }^{94}$, Q. Luce ${ }^{39}$, J.P. Lundquist ${ }^{76}$, A. Machado Payeras $^{22}$, G. Mancarella ${ }^{56,48}$, D. Mandat ${ }^{32}$, B.C. Manning ${ }^{13}$, J. Manshanden ${ }^{43}$, P. Mantsch ${ }^{a}$, S. Marafico ${ }^{34}$, A.G. Mariazzi ${ }^{4}$, I.C. Mariş ${ }^{14}$, G. Marsella ${ }^{61,47}$, D. Martello ${ }^{56,48}$, S. Martinelli ${ }^{41,8}$, O. Martínez Bravo ${ }^{64}$, M. Mastrodicasa ${ }^{57,46}$, H.J. Mathes ${ }^{41}$, J. Matthews ${ }^{88}$, G. Matthiae ${ }^{62,51}$, E. Mayotte ${ }^{38}$, P.O. Mazur ${ }^{a}$, G. MedinaTanco $^{68}$, D. Melo $^{8}$, A. Menshikov ${ }^{40}$, K.-D. Merenda ${ }^{86}$, S. Michal ${ }^{33}$, M.I. Micheletti ${ }^{6}$, L. Miramonti ${ }^{59,49}$, S. Mollerach ${ }^{1}$, F. Montanet ${ }^{36}$, C. Morello ${ }^{54,52}$, M. Mostafá ${ }^{91}$, A.L. Müller ${ }^{8}$, M.A. Muller ${ }^{22}$, K. Mulrey ${ }^{15}$, R. Mussa ${ }^{52}$, M. Muzio ${ }^{90}$, W.M. Namasaka ${ }^{38}$, A. Nasr-Esfahani ${ }^{38}$, L. Nellen ${ }^{68}$, M. Niculescu-Oglinzanu ${ }^{73}$, M. Niechciol ${ }^{44}$, D. Nitz ${ }^{89}$, D. Nosek ${ }^{31}$, V. Novotny ${ }^{31}$, L. Nožka ${ }^{33}$, A Nucita ${ }^{56,48}$, L.A. Núñez ${ }^{30}$, M. Palatka ${ }^{32}$, J. Pallotta ${ }^{2}$, P. Papenbreer ${ }^{38}$, G. Parente ${ }^{79}$, A. Parra ${ }^{64}$, J. Pawlowsky ${ }^{38}$, M. Pech ${ }^{32}$, F. Pedreira ${ }^{79}$, J. Pȩkala ${ }^{70}$, R. Pelayo ${ }^{65}$, J. Peña-Rodriguez ${ }^{30}$, E.E. Pereira Martins ${ }^{39,8}$, J. Perez Armand ${ }^{21}$, C. Pérez Bertolli $^{8,41}$, M. Perlin ${ }^{8,41}$, L. Perrone ${ }^{56,48}$, S. Petrera ${ }^{45,46}$, T. Pierog ${ }^{41}$, M. Pimenta ${ }^{72}$, V. Pirronello ${ }^{58,47}$, M. Platino ${ }^{8}$, B. Pont $^{80}$, M. Pothast ${ }^{81,80}$, P. Privitera ${ }^{92}$, M. Prouza ${ }^{32}$, A. Puyleart ${ }^{89}$, S. Querchfeld ${ }^{38}$, J. Rautenberg ${ }^{38}$, D. Ravignani ${ }^{8}$, M. Reininghaus ${ }^{41,8}$, J. Ridky ${ }^{32}$, F. Riehn ${ }^{72}$, M. Risse ${ }^{44}$, V. Rizi ${ }^{57,46}$, W. Rodrigues de Carvalho ${ }^{21}$, J. Rodriguez Rojo ${ }^{11}$, M.J. Roncoroni ${ }^{8}$, S. Rossoni ${ }^{43}$, M. Roth ${ }^{41}$, E. Roulet ${ }^{1}$, A.C. Rovero ${ }^{5}$, P. Ruehl ${ }^{44}$, A. Saftoiu ${ }^{73}$, F. Salamida ${ }^{57,46}$, H. Salazar ${ }^{64}$, G. Salina ${ }^{51}$, J.D. Sanabria Gomez ${ }^{30}$, F. Sánchez ${ }^{8}$, E.M. Santos ${ }^{21}$, E. Santos ${ }^{32}$, F. Sarazin ${ }^{86}$, R. Sarmento ${ }^{72}$, C. Sarmiento-Cano ${ }^{8}$, R. Sato ${ }^{11}$, 
P. Savina ${ }^{56,48,34,94}$, C.M. Schäfer ${ }^{41}$, V. Scherini ${ }^{56,48}$, H. Schieler ${ }^{41}$, M. Schimassek ${ }^{39,8}$, M. Schimp ${ }^{38}$, F. Schlüter ${ }^{41,8}$, D. Schmidt ${ }^{39}$, O. Scholten ${ }^{84,15}$, P. Schovánek ${ }^{32}$, F.G. Schröder ${ }^{93,41}$, S. Schröder ${ }^{38}$, J. Schulte ${ }^{42}$, S.J. Sciutto ${ }^{4}$, M. Scornavacche ${ }^{8,41}$, A. Segreto ${ }^{53,47}$, S. Sehgal ${ }^{38}$, R.C. Shellard ${ }^{16}$, G. Sigl ${ }^{43}$, G. Silli ${ }^{8,41}$, O. Sima ${ }^{73, f}$, R. Šmída ${ }^{92}$, P. Sommers ${ }^{91}$, J.F. Soriano ${ }^{87}$, J. Souchard ${ }^{36}$, R. Squartini ${ }^{10}$, M. Stadelmaier ${ }^{41,8}$, D. Stanca ${ }^{73}$, S. Stanič ${ }^{76}$, J. Stasielak ${ }^{70}$, P. Stassi ${ }^{36}$, A. Streich ${ }^{39,8}$, M. Suárez-Durán ${ }^{14}$, T. Sudholz ${ }^{13}$, T. Suomijärvi ${ }^{37}$, A.D. Supanitsky ${ }^{8}$, Z. Szadkowski ${ }^{71}$, A. Tapia ${ }^{29}$, C. Taricco ${ }^{63,52}$, C. Timmermans ${ }^{81,80}$, O. Tkachenko ${ }^{41}$, P. Tobiska ${ }^{32}$, C.J. Todero Peixoto ${ }^{19}$, B. Tomé ${ }^{72}$, Z. Torrès ${ }^{36}$, A. Travaini ${ }^{10}$, P. Travnicek $^{32}$, C. Trimarelli ${ }^{57,46}$, M. Tueros ${ }^{4}$, R. Ulrich ${ }^{41}$, M. Unger ${ }^{41}$, L. Vaclavek ${ }^{33}$, M. Vacula ${ }^{33}$, J.F. Valdés Galicia ${ }^{68}$, L. Valore ${ }^{60,50}$, E. Varela ${ }^{64}$, A. Vásquez-Ramírez ${ }^{30}$, D. Veberič ${ }^{41}$, C. Ventura ${ }^{27}$, I.D. Vergara Quispe ${ }^{4}$, V. Verzi ${ }^{51}$, J. Vicha ${ }^{32}$, J. Vink $^{83}$, S. Vorobiov ${ }^{76}$, H. Wahlberg ${ }^{4}$, C. Watanabe ${ }^{26}$, A.A. Watson ${ }^{c}$, M. Weber ${ }^{40}$, A. Weindl ${ }^{41}$, L. Wiencke ${ }^{86}$, H. Wilczyński ${ }^{70}$, M. Wirtz ${ }^{42}$, D. Wittkowski ${ }^{38}$, B. Wundheiler ${ }^{8}$, A. Yushkov $^{32}$, O. Zapparrata ${ }^{14}$, E. Zas ${ }^{79}$, D. Zavrtanik ${ }^{76,77}$, M. Zavrtanik ${ }^{77,76}$, L. Zehrer ${ }^{76}$

${ }^{1}$ Centro Atómico Bariloche and Instituto Balseiro (CNEA-UNCuyo-CONICET), San Carlos de Bariloche, Argentina

${ }^{2}$ Centro de Investigaciones en Láseres y Aplicaciones, CITEDEF and CONICET, Villa Martelli, Argentina

${ }^{3}$ Departamento de Física and Departamento de Ciencias de la Atmósfera y los Océanos, FCEyN, Universidad de Buenos Aires and CONICET, Buenos Aires, Argentina

${ }^{4}$ IFLP, Universidad Nacional de La Plata and CONICET, La Plata, Argentina

${ }^{5}$ Instituto de Astronomía y Física del Espacio (IAFE, CONICET-UBA), Buenos Aires, Argentina

${ }^{6}$ Instituto de Física de Rosario (IFIR) - CONICET/U.N.R. and Facultad de Ciencias Bioquímicas y Farmacéuticas U.N.R., Rosario, Argentina

${ }^{7}$ Instituto de Tecnologías en Detección y Astropartículas (CNEA, CONICET, UNSAM), and Universidad Tecnológica Nacional - Facultad Regional Mendoza (CONICET/CNEA), Mendoza, Argentina

${ }^{8}$ Instituto de Tecnologías en Detección y Astropartículas (CNEA, CONICET, UNSAM), Buenos Aires, Argentina

${ }^{9}$ International Center of Advanced Studies and Instituto de Ciencias Físicas, ECyT-UNSAM and CONICET, Campus Miguelete - San Martín, Buenos Aires, Argentina

${ }^{10}$ Observatorio Pierre Auger, Malargüe, Argentina

${ }^{11}$ Observatorio Pierre Auger and Comisión Nacional de Energía Atómica, Malargüe, Argentina

12 Universidad Tecnológica Nacional - Facultad Regional Buenos Aires, Buenos Aires, Argentina

${ }^{13}$ University of Adelaide, Adelaide, S.A., Australia

14 Université Libre de Bruxelles (ULB), Brussels, Belgium

15 Vrije Universiteit Brussels, Brussels, Belgium

${ }^{16}$ Centro Brasileiro de Pesquisas Fisicas, Rio de Janeiro, RJ, Brazil

${ }^{17}$ Centro Federal de Educação Tecnológica Celso Suckow da Fonseca, Nova Friburgo, Brazil

${ }^{18}$ Instituto Federal de Educação, Ciência e Tecnologia do Rio de Janeiro (IFRJ), Brazil

${ }^{19}$ Universidade de São Paulo, Escola de Engenharia de Lorena, Lorena, SP, Brazil

${ }^{20}$ Universidade de São Paulo, Instituto de Física de São Carlos, São Carlos, SP, Brazil

${ }^{21}$ Universidade de São Paulo, Instituto de Física, São Paulo, SP, Brazil

${ }^{22}$ Universidade Estadual de Campinas, IFGW, Campinas, SP, Brazil

${ }^{23}$ Universidade Estadual de Feira de Santana, Feira de Santana, Brazil

${ }^{24}$ Universidade Federal do ABC, Santo André, SP, Brazil

${ }^{25}$ Universidade Federal do Paraná, Setor Palotina, Palotina, Brazil

${ }^{26}$ Universidade Federal do Rio de Janeiro, Instituto de Física, Rio de Janeiro, RJ, Brazil

${ }^{27}$ Universidade Federal do Rio de Janeiro (UFRJ), Observatório do Valongo, Rio de Janeiro, RJ, Brazil

${ }^{28}$ Universidade Federal Fluminense, EEIMVR, Volta Redonda, RJ, Brazil

${ }^{29}$ Universidad de Medellín, Medellín, Colombia

${ }^{30}$ Universidad Industrial de Santander, Bucaramanga, Colombia

${ }^{31}$ Charles University, Faculty of Mathematics and Physics, Institute of Particle and Nuclear Physics, Prague, Czech Republic

32 Institute of Physics of the Czech Academy of Sciences, Prague, Czech Republic 
${ }^{33}$ Palacky University, RCPTM, Olomouc, Czech Republic

34 CNRS/IN2P3, IJCLab, Université Paris-Saclay, Orsay, France

${ }^{35}$ Laboratoire de Physique Nucléaire et de Hautes Energies (LPNHE), Sorbonne Université, Université de Paris, CNRSIN2P3, Paris, France

${ }^{36}$ Univ. Grenoble Alpes, CNRS, Grenoble Institute of Engineering Univ. Grenoble Alpes, LPSC-IN2P3, 38000 Grenoble, France

${ }^{37}$ Université Paris-Saclay, CNRS/IN2P3, IJCLab, Orsay, France

38 Bergische Universität Wuppertal, Department of Physics, Wuppertal, Germany

${ }^{39}$ Karlsruhe Institute of Technology (KIT), Institute for Experimental Particle Physics, Karlsruhe, Germany

${ }^{40}$ Karlsruhe Institute of Technology (KIT), Institut für Prozessdatenverarbeitung und Elektronik, Karlsruhe, Germany

${ }^{41}$ Karlsruhe Institute of Technology (KIT), Institute for Astroparticle Physics, Karlsruhe, Germany

${ }^{42}$ RWTH Aachen University, III. Physikalisches Institut A, Aachen, Germany

${ }^{43}$ Universität Hamburg, II. Institut für Theoretische Physik, Hamburg, Germany

${ }^{44}$ Universität Siegen, Department Physik - Experimentelle Teilchenphysik, Siegen, Germany

${ }^{45}$ Gran Sasso Science Institute, L'Aquila, Italy

46 INFN Laboratori Nazionali del Gran Sasso, Assergi (L'Aquila), Italy

${ }^{47}$ INFN, Sezione di Catania, Catania, Italy

${ }^{48}$ INFN, Sezione di Lecce, Lecce, Italy

${ }^{49}$ INFN, Sezione di Milano, Milano, Italy

${ }^{50}$ INFN, Sezione di Napoli, Napoli, Italy

${ }^{51}$ INFN, Sezione di Roma "Tor Vergata", Roma, Italy

52 INFN, Sezione di Torino, Torino, Italy

53 Istituto di Astrofisica Spaziale e Fisica Cosmica di Palermo (INAF), Palermo, Italy

54 Osservatorio Astrofisico di Torino (INAF), Torino, Italy

55 Politecnico di Milano, Dipartimento di Scienze e Tecnologie Aerospaziali, Milano, Italy

${ }^{56}$ Università del Salento, Dipartimento di Matematica e Fisica "E. De Giorgi”, Lecce, Italy

57 Università dell'Aquila, Dipartimento di Scienze Fisiche e Chimiche, L'Aquila, Italy

58 Università di Catania, Dipartimento di Fisica e Astronomia, Catania, Italy

${ }^{59}$ Università di Milano, Dipartimento di Fisica, Milano, Italy

${ }^{60}$ Università di Napoli "Federico II", Dipartimento di Fisica "Ettore Pancini”, Napoli, Italy

${ }^{61}$ Università di Palermo, Dipartimento di Fisica e Chimica "E. Segrè", Palermo, Italy

62 Università di Roma "Tor Vergata", Dipartimento di Fisica, Roma, Italy

${ }^{63}$ Università Torino, Dipartimento di Fisica, Torino, Italy

${ }^{64}$ Benemérita Universidad Autónoma de Puebla, Puebla, México

${ }^{65}$ Unidad Profesional Interdisciplinaria en Ingeniería y Tecnologías Avanzadas del Instituto Politécnico Nacional (UPIITA-IPN), México, D.F., México

66 Universidad Autónoma de Chiapas, Tuxtla Gutiérrez, Chiapas, México

${ }^{67}$ Universidad Michoacana de San Nicolás de Hidalgo, Morelia, Michoacán, México

${ }^{68}$ Universidad Nacional Autónoma de México, México, D.F., México

${ }^{69}$ Universidad Nacional de San Agustin de Arequipa, Facultad de Ciencias Naturales y Formales, Arequipa, Peru

${ }^{70}$ Institute of Nuclear Physics PAN, Krakow, Poland

${ }^{71}$ University of Łódź, Faculty of High-Energy Astrophysics, Łódź, Poland

${ }^{72}$ Laboratório de Instrumentação e Física Experimental de Partículas - LIP and Instituto Superior Técnico - IST, Universidade de Lisboa - UL, Lisboa, Portugal

73 "Horia Hulubei” National Institute for Physics and Nuclear Engineering, Bucharest-Magurele, Romania

${ }^{74}$ Institute of Space Science, Bucharest-Magurele, Romania

75 University Politehnica of Bucharest, Bucharest, Romania

76 Center for Astrophysics and Cosmology (CAC), University of Nova Gorica, Nova Gorica, Slovenia

${ }^{77}$ Experimental Particle Physics Department, J. Stefan Institute, Ljubljana, Slovenia

78 Universidad de Granada and C.A.F.P.E., Granada, Spain

${ }^{79}$ Instituto Galego de Física de Altas Enerxías (IGFAE), Universidade de Santiago de Compostela, Santiago de Compostela, Spain 
${ }^{80}$ IMAPP, Radboud University Nijmegen, Nijmegen, The Netherlands

${ }^{81}$ Nationaal Instituut voor Kernfysica en Hoge Energie Fysica (NIKHEF), Science Park, Amsterdam, The Netherlands

82 Stichting Astronomisch Onderzoek in Nederland (ASTRON), Dwingeloo, The Netherlands

${ }^{83}$ Universiteit van Amsterdam, Faculty of Science, Amsterdam, The Netherlands

${ }^{84}$ University of Groningen, Kapteyn Astronomical Institute, Groningen, The Netherlands

85 Case Western Reserve University, Cleveland, OH, USA

86 Colorado School of Mines, Golden, CO, USA

${ }^{87}$ Department of Physics and Astronomy, Lehman College, City University of New York, Bronx, NY, USA

${ }^{88}$ Louisiana State University, Baton Rouge, LA, USA

${ }^{89}$ Michigan Technological University, Houghton, MI, USA

${ }^{90}$ New York University, New York, NY, USA

${ }^{91}$ Pennsylvania State University, University Park, PA, USA

92 University of Chicago, Enrico Fermi Institute, Chicago, IL, USA

93 University of Delaware, Department of Physics and Astronomy, Bartol Research Institute, Newark, DE, USA

94 University of Wisconsin-Madison, Department of Physics and WIPAC, Madison, WI, USA

${ }^{a}$ Fermi National Accelerator Laboratory, Fermilab, Batavia, IL, USA

${ }^{b}$ Max-Planck-Institut für Radioastronomie, Bonn, Germany

${ }^{c}$ School of Physics and Astronomy, University of Leeds, Leeds, United Kingdom

${ }^{d}$ Colorado State University, Fort Collins, CO, USA

${ }^{e}$ now at Hakubi Center for Advanced Research and Graduate School of Science, Kyoto University, Kyoto, Japan

$f$ also at University of Bucharest, Physics Department, Bucharest, Romania 